\title{
THE CLASS OF ALL $S$-PREGROUPS IS NOT FINITELY AXIOMATIZABLE
}

\author{
DEKO V. DEKOV
}

(Communicated by Warren J. Wong)

\begin{abstract}
In order to investigate the amalgamated free products of groups, in 1950 R. Baer (Free sums of groups and their generalizations. II, Amer. J. Math. 72 (1950), 625-646) introduced the concept of an $S$-pregroup and gave an infinite set of elementary (i.e., of a first-order language) axioms for $S$-pregroups. The term " $S$-pregroup" was introduced by J. R. Stallings (Adian groups and pregroups, Essays in Group Theory, Math. Sci. Res. Inst. Publ., vol. 8, Springer-Verlag, New York, 1987, pp. 321-342), who suggested the problem of finding a finite set of elementary axioms for $S$-pregroups (ibid, Question 5, The first part, p. 340). In the present paper we show that the class of all $S$-pregroups is not finitely axiomatizable, i.e., it cannot be characterized by any finite set of elementary axioms.
\end{abstract}

\section{Preliminaries}

In this section we review some of R. Baer's results on $S$-pregroups.

A partial groupoid is a triple $G=(G, D, \mu)$ where $G$ is a nonempty set, $D \subseteq G \times G$ and $\mu: D \rightarrow G$ is a mapping. We use the following notations [5, 4]:

$$
\begin{gathered}
x \cdot y=\mu(x, y), \\
(x, y)_{D} \text { iff }(x, y) \in D .
\end{gathered}
$$

Let $G=(G, D, \mu)$ be a partial groupoid and $F(G)$ be the free group on the set $G$. Let $\theta_{0}$ be the relation on $F(G)$ defined as follows: $(x \cdot y, x y) \in \theta_{0}$ iff $(x, y)_{D}$. Let $\theta$ be the congruence on $F(G)$ generated by $\theta_{0}$. Then $U(G)=$ $F(G) / \theta$ is the universal group of the partial groupoid $G$.

Definition. A partial groupoid $G=(G, D, \mu)$ is said to be an $S$-pregroup if it satisfies the following axioms:

(A1) There exists an identity element $1 \in G$ such that for all $x \in G$, $(x, 1)_{D},(1, x)_{D}$, and $x \cdot 1=1 \cdot x=x$.

(A2) For each $x \in G$, there exists $x^{-1} \in G$ such that $\left(x, x^{-1}\right)_{D},\left(x^{-1}, x\right)_{D}$, and $x \cdot x^{-1}=x^{-1} \cdot x=1$.

(A3) For all $x, y, z \in G$,

(i) if $(x, y)_{D}$ and $(y, z)_{D}$, then: $(x \cdot y, z)_{D}$ iff $(x, y \cdot z)_{D}$.

(ii) if $(x, y)_{D},(y, z)_{D}$, and $(x \cdot y, z)_{D}$, then $x \cdot(y \cdot z)=(x \cdot y) \cdot z$.

Received by the editors December 11, 1990 and, in revised form, January 7, 1991. 1991 Mathematics Subject Classification. Primary 20 A 15. 
(A4) For all $x, y \in G, x \sim y(\bmod \theta)$ implies $x=y$.

(A5) For each reduced word $X, 1 \sim X(\bmod \theta)$ implies $1=X$.

Axiom (A4) means that $G$ embeds in its universal group. Axiom (A5) means that in the universal group of $G$ the identity element cannot be represented as a nontrivial reduced word.

Theorem 1 (R. Baer [1, §4, Theorem]). A partial groupoid $G=(G, D, \mu)$ is an $S$-pregroup if and only if it satisfies axioms (A1), (A2), (A3), and for each $n \geq 4$, the following axiom:

$\left(S_{n}\right)$ For all $x_{1}, \ldots, x_{n} \in G$, if for $i=1, \ldots, n-1,\left(x_{i}, x_{i+1}^{-1}\right)_{D}$ and $\left(x_{n}, x_{1}^{-1}\right)_{D}$, then there exists $j \leq n-2$ such that $\left(x_{j}, x_{j+2}^{-1}\right)_{D}$.

It is routine to verify that the infinite set of axioms for $S$-pregroups due to R. Baer can be written as an infinite set of elementary axioms.

\section{The ClASS OF ALL $S$-PREgROUPS IS NOT FINITELY AXIOMATIZABLE}

We will prove that the infinite set of elementary axioms for $S$-pregroups due to R. Baer is not equivalent to any of its finite subsets. From this follows the fact that the class of all $S$-pregroups is not finitely axiomatizable. The reader is referred to [2] for the fundamentals of first-order theories.

Theorem 2. The class of all $S$-pregroups is not finitely axiomatizable.

Proof. By Theorem 1, a partial groupoid $G$ is an $S$-pregroup if and only if it satisfies axioms (A1), (A2), (A3), and for each $n \geq 4$, axiom $\left(S_{n}\right)$. We will prove that (A1), (A2), (A3), and $\left(S_{k}\right)$ for $k=4,5, \ldots, n-1$ do not imply $\left(S_{n}\right)$. To prove this we construct a partial groupoid $G_{n}$ that satisfies (A1), (A2), (A3), and $\left(S_{k}\right)$ for $k=4,5, \ldots, n-1$ but not $\left(S_{n}\right)$.

Let $G_{n}=\left(G_{n}, D, \mu\right)$. We define

$$
G_{n}=\left\{1, a_{0}, \ldots, a_{n-1}, a_{0}^{-1}, \ldots, a_{n-1}^{-1}, b_{0}, \ldots, b_{n-1}, b_{0}^{-1}, \ldots, b_{n-1}^{-1}\right\} \text {. }
$$

We will denote by $\oplus$ addition modulo $n$. Let $i=0,1, \ldots, n-1$. We define partial multiplication in $G_{n}$ as follows: 1 is the identity of $G_{n}$ and $a_{i}^{-1}\left[b_{i}^{-1}\right]$ is the inverse of $a_{i}\left[b_{i}\right]$. All other products are

$$
\begin{aligned}
b_{i} \cdot a_{i \oplus 1}=a_{i}, & b_{i}^{-1} \cdot a_{i}=a_{i \oplus 1}, & a_{i} \cdot a_{i \oplus 1}^{-1}=b_{i}, \\
a_{i \oplus 1}^{-1} \cdot b_{i}^{-1}=a_{i}^{-1}, & a_{i}^{-1} \cdot b_{i}=a_{i \oplus 1}^{-1}, & a_{i \oplus 1} \cdot a_{i}^{-1}=b_{i}^{-1} .
\end{aligned}
$$

Clearly, $G_{n}$ satisfies (A1) and (A2). It is routine to verify that $G_{n}$ satisfies (A3). We will prove that $G_{n}$ satisfies $\left(S_{k}\right)$ for $k=4,5, \ldots, n-1$.

Let $X=x_{1} \cdots x_{m}$ be a word. A subword of $X$ is a word $x_{r} x_{r+1} \cdots x_{s}$, where $1 \leq r \leq s \leq m$. The subword $x_{1} \cdots x_{s}$ (i.e., $r=1$ ) of $X$ is called a left factor of $X$ [3], and the element $x_{1}$ is called the first letter of $X$. We will say that the word $x_{1} \cdots x_{m}$ is regular if for $i=1, \ldots, m-1,\left(x_{i}, x_{i+1}^{-1}\right)_{D}$ and $\left(x_{m}, x_{1}^{-1}\right)_{D}$. Thus $\left(S_{m}\right), m \geq 4$, holds iff for every regular word $x_{1} \cdots x_{m}$ there exists $j \leq m-2$ such that $\left(x_{j}, x_{j+2}^{-1}\right)_{D}$.

We will consider regular words of length $k$, where $4 \leq k \leq n-1$.

If in a regular word $X=x_{1} \cdots x_{k}$, we have $x_{i}=1$ for some $i \leq k$ or $x_{j}=x_{j+1}$ for some $j \leq k-1$, then, obviously, $X$ satisfies $\left(S_{k}\right)$. In what follows we will omit these cases. 
For $x \in G_{n}$ we define

$$
C(x)=\left\{y:\left(x, y^{-1}\right)_{D}, y \neq 1, \text { and } y \neq x\right\} .
$$

For $i=0,1, \ldots, n-1$, we have

$$
\begin{gathered}
C\left(a_{i}\right)=\left\{a_{i \ominus 1}, a_{i \oplus 1}\right\}, \\
C\left(a_{i}^{-1}\right)=\left\{b_{i \ominus 1}, b_{i}^{-1}\right\}, \\
C\left(b_{i}\right)=\left\{a_{i \oplus 1}^{-1}\right\}, \\
C\left(b_{i}^{-1}\right)=\left\{a_{i}^{-1}\right\} .
\end{gathered}
$$

We distinguish four cases.

Case 1. The first letter of a regular word $X$ is $a_{i}$. Using (1) we see that $X$ has as a subword at least one of the following words:

$$
a_{j} a_{j \ominus 1} a_{j} \text { or } a_{j} a_{j \oplus 1} a_{j}, \quad \text { where } j \in\{0,1, \ldots, n-1\} .
$$

Thus $\left(S_{k}\right)$ holds.

Case 2. The first letter of a regular word $X$ is $a_{i}^{-1}$. Using (2), (3), and (4) we see that $X$ has as a left factor, either

$$
a_{i}^{-1} b_{i \ominus 1} a_{i}^{-1} \text { or } a_{i}^{-1} b_{i}^{-1} a_{i}^{-1} \text {. }
$$

Thus $\left(S_{k}\right)$ holds.

Case 3. The first letter of a regular word $X$ is $b_{i}$. Using (3), (2), and (4) we see that $X$ has as a left factor, either

$$
b_{i} a_{i \oplus 1}^{-1} b_{i} \quad \text { or } \quad b_{i} a_{i \oplus 1}^{-1} b_{i \oplus 1}^{-1} a_{i \oplus 1}^{-1} .
$$

Thus $\left(S_{k}\right)$ holds.

Case 4. The first letter of a regular word $X$ is $b_{i}^{-1}$. Using (4), (2), and (3) we see that $X$ has as a left factor, either

$$
b_{i}^{-1} a_{i}^{-1} b_{i \ominus 1} a_{i}^{-1} \text { or } b_{i}^{-1} a_{i}^{-1} b_{i}^{-1} \text {. }
$$

Thus $\left(S_{k}\right)$ holds.

We have proved that $G_{n}$ satisfies $\left(S_{k}\right)$ for $k=4,5, \ldots, n-1$. To show that $G_{n}$ does not satisfy $\left(S_{n}\right)$, note that the word $a_{0} a_{1} \cdots a_{n-1}$ is regular, but for $j=0,1, \ldots, n-3,\left(a_{j}, a_{j+2}^{-1}\right) \notin D$. This completes the proof of Theorem 2.

\section{REFERENCES}

1. R. Baer, Free sums of groups and their generalizations. II, Amer. J. Math. 72 (1950), 625646.

2. J. Barwise, ed., Handbook of mathematical logic, North-Holland, Amsterdam, New York, and Oxford, 1977.

3. G. Lallement, Semigroups and combinatorial applications, Wiley, New York, 1979.

4. F. Rimlinger, Pregroups and Bass-Serre theory, Mem. Amer. Math. Soc., no. 361, Amer. Math. Soc., Providence, RI, 1987.

5. J. R. Stallings, Adian groups and pregroups, Essays in Group Theory, Math. Sci. Res. Inst. Publ., vol. 8, Springer-Verlag, New York, 1987, pp. 321-342. 